\title{
Sélection des variétés locales et techniques de culture du figuier en Israël
}

Raphaël Assaf

Département d'Arboriculture fruitière,

Agricultural Research

Organisation (ARO),

Centre de recherches

Néwé-Ya'ar,

PO Box 1021,

Ramat Yishay 30095, Israël

mata@netvision.net.il

\section{Local fig clonal selections and cultivation techniques in Israel.}

Abstract - Introduction. In Israel, the fig is very demanded by the consumer. This article describes the new techniques developed over the last 50 years for the cultivation of the fig tree and presents varietal test results carried out in number of orchards over successive plots. The study covers a great number of varieties resulting from a vast collection throughout Israel. General. After an overview of the fig tree origin, the characteristics of the flower and its fecundation, or caprification, by Blastophaga psenes are given. The fig tree can be uniferous and produce only one harvest in autumn, or biferous and bear fruit twice: in spring (fig-flowers), then in summer and autumn (figs of autumn). The mechanisms of the tree growth and fruiting are briefly presented. Materials and methods. The study carried out related on the production of experimental orchards set up in Upper Galilee (Israel) and to the observation of the production of fig trees collected throughout Israël, Gaza, Sinai and the Golan. Results and classification of the studied varieties. The classification of the varieties of fig trees initially takes into account the basic characters of the fruit, then additional observations. Four groups of fig trees were distinguished from the green-yellow, green-purple, brown striated and black color of their fruit. Each variety identified in these groups was described. Tree growth was analyzed and the agricultural economics of the fig tree was considered. The techniques most adapted for the fig production, from tree plantation to harvest, are presented. Conclusions and discussion. Research carried out showed that certain fig tree types shared out in the four groups identified are particularly recommended. The Nazareth variety is recommended for the production of fig-flowers with the agrotechnics.

Israel / Ficus carica / selection / varieties / cultivation

Sélection des variétés locales et techniques de culture du figuier en Israël.

Résumé - Introduction. En Israël, la figue est un fruit très demandé par le consommateur. L'article fait le point des nouvelles techniques testées et adoptées pour la culture du figuier et présente les résultats d'essais variétaux effectués pendant 50 ans sur des vergers successifs en stations expérimentales, constitués d'un grand nombre de variétés issues d'une vaste collecte à travers Israël. Généralités. Après un rappel de l'origine du figuier, les particularités de la fleur et de sa fécondation, ou caprification, par Blastophaga psenes sont évoquées. Le figuier peut être unifère et produire une seule récolte en automne, ou bifère et fructifier deux fois : au printemps (figues-fleurs), puis en été et en automne (figues d'automne). Les mécanismes de la croissance et de la fructification de l'arbre sont présentés brièvement. Matériel et méthodes. L'étude réalisée a porté sur la production des vergers expérimentaux mis en place en Haute Galilée (Israël) et sur l'observation de la production de figuiers collectés dans l'ensemble du pays, Gaza, Sinaï et le Golan. Résultats et classification des variétés étudiées. La classification des variétés de figuiers repose d'abord sur les caractères de base du fruit, puis sur des observations annexes. Globalement, quatre groupes de figuiers ont été distingués à partir de la couleur vert-jaune, vert-violet, brun strié et noire de leurs fruits. Chacune des variétés identifiées dans ces groupes a été décrite, la croissance des arbres a été analysée et l'économie agricole du figuier a été considérée. Les techniques les plus appropriées pour la conduite du figuier, de la plantation à la récolte, sont exposées. Conclusions et discussion. À l'issue des travaux effectués, certains types de figuiers répartis dans les quatre groupes identifiés sont plus particulièrement recommandés. La variété Nazareth est préconisée pour la production de figues-fleurs à l'aide des techniques agronomiques présentées.

Israël / Ficus carica / sélection / variété / pratique culturale
Fruits, 2001, vol. 56, p. 101-12 (C) 2001 Cirad/EDP Sciences All rights reserved

RESUMEN EsPañol, p. 120 


\section{Introduction}

Le figuier existe dans tous les pays méditerranéens depuis des milliers d'années. C'est un arbre très commun en Israël où il pousse et fructifie dans toutes les zones climatiques du pays. Depuis l'Antiquité, il y occupe une place très importante. Il est cité dans la Bible parmi les sept fruits qui prolifèrent sur ces terres. La figue est très appréciée de la population, même de celle originaire du nord de l'Europe. La demande sur les marchés est grande et le consommateur est prêt à payer un prix élevé pour ce fruit considéré comme exotique.

Avant la création de l'État d'Israël en 1948, le figuier occupait environ 12350 ha [1]. Aujourd'hui, à côté des vergers en culture intensive qui sont devenus moins nombreux (environ $40 \mathrm{ha}$ ), il existe beaucoup de bosquets d'arbres dans les anciens vergers abandonnés, les cours des maisons, le long des routes et des rivières ; certains sont aussi éparpillés dans les forêts. Dans tout le bassin méditerranéen, le figuier est considéré comme une plante spontanée. Aujourd'hui, il a une importance économique qui pourrait paraître marginale par rapport à celle d'autres espèces fruitières, mais son potentiel de développement paraît important à la condition d'appliquer des techniques agronomiques performantes à des variétés à grands rendements et fruits de qualité. Il serait alors possible de mieux valoriser la figue, d'obtenir une récolte plus groupée, moins coûteuse et de disposer d'un fruit moins fragile, se conservant relativement bien.

Cet article fait le point de nouvelles techniques testées et adoptées pour la culture du figuier et présente les résultats d'essais variétaux effectués pendant 50 ans sur des vergers successifs en stations expérimentales, constitués d'un grand nombre de variétés issues d'une vaste collecte à travers Israël.

\section{Généralités}

\section{1. Origine du figuier}

Le figuier domestique, Ficus carica L, est un arbre à feuilles caduques de la famille des Moracées. Son nom Ficus vient de l'hébreu «Faga »utilisé pour désigner une figue qui n'est pas mûre. La plante serait originaire du Proche Orient, bien que l'emplacement exact soit difficile à localiser. Selon certains botanistes, son centre d'origine serait le Yémen d'où il aurait été diffusé en Israël, en Syrie, puis dans tout le Bassin méditerranéen [1]. Les figuiers d'Israël sont mentionnés dans des inscriptions égyptiennes datant de 2375 av. J.-C. Par ailleurs, des restes de fruits datant de 7000 ans av. J.-C ont été trouvés dans les fouilles de Jéricho. De Candolle, décrivant la culture du figuier, a signalé qu'il se trouvait à l'état sauvage ou demi-sauvage sur une très grande région dont le centre serait la Syrie et Israël [1-3]. D'après une description de la flore de la Syrie et de la Palestine due à Post [1], il y aurait en Israël plusieurs figuiers sauvages tels que Ficus carica var. genuina Boiss, Ficus carica var. riparium Hausk, ainsi que les variétés rupestris Hausk, globosa Boiss et pseudo-sycomorus.

\subsection{La figue}

La figue est un sycone, réceptacle charnu et creux, présentant à son extrémité une ouverture, ou ostiole, bordée intérieurement par des bractées étroitement imbriquées. La cavité intérieure est tapissée par les organes de la reproduction sexuée qui peuvent être de trois types : femelles, mâles ou mâles stériles.

Les fleurs femelles ont un ovaire uniovulé et les fleurs mâles peuvent, dans certains cas, porter des étamines. Les fleurs femelles fécondées donnent des graines ou reçoivent la ponte des Blastophagas et évoluent en galles [1-5]. La fécondation du figuier, dénommée «caprification »est très complexe. Elle s'effectue grâce à Blastophaga psenes, insecte qui se développe dans le fruit du caprifiguier (figuier mâle, sauvage en général) et en sort en se couvrant de pollen lors de son passage par l'orifice de l'ostiole, zone des fleurs mâles. Cette opération très connue a été décrite en détails depuis des temps très anciens [1-5]. La longueur du style de la fleur femelle est déterminante pour assurer le succès de la ponte : alors qu'il existe des figuiers femelles, ou domestiques, à fleurs à longistyles et 


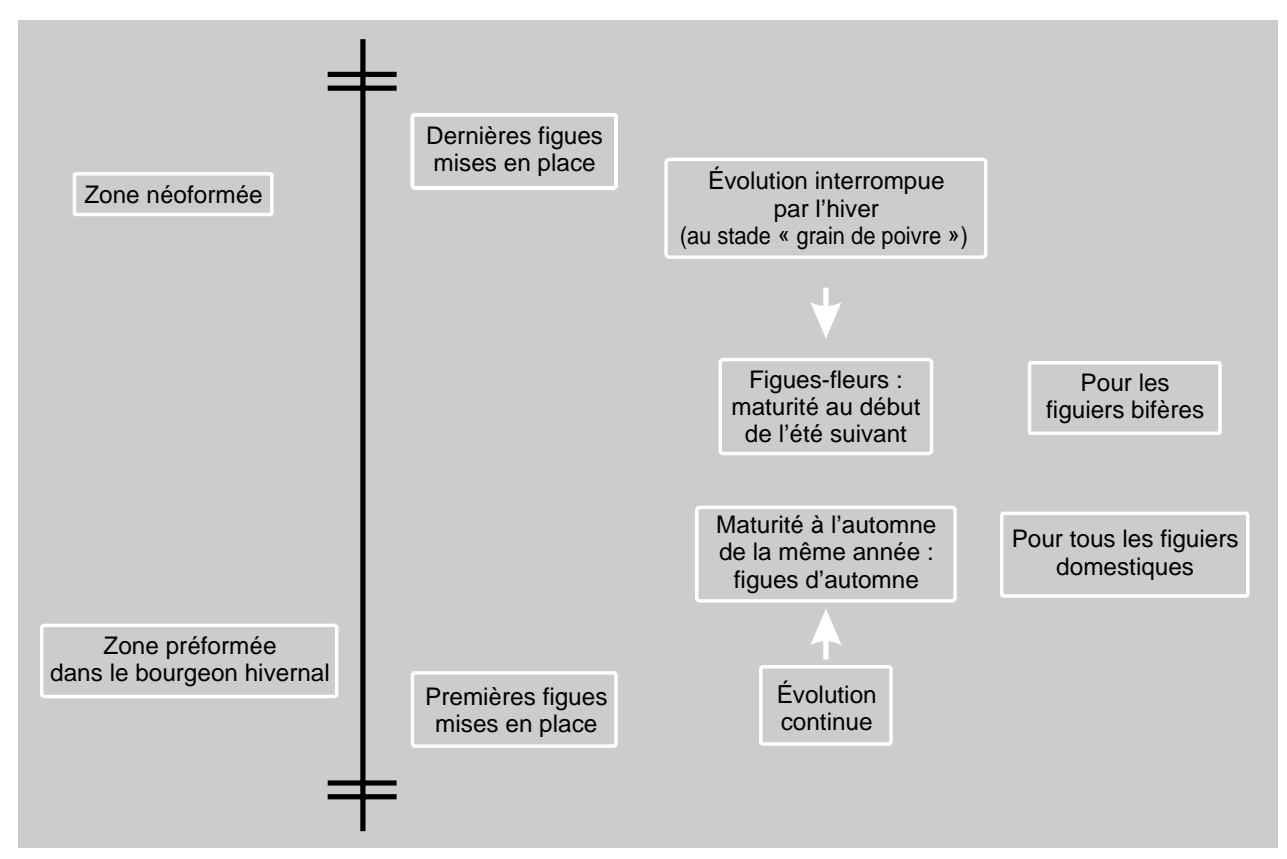

fleurs mâles stériles, et des figuiers mâles avec des fleurs femelles brévistyles et fleurs mâles fertiles, la ponte du Blastophaga n'est possible que dans des fleurs brévistyles, donc mâles, que l'ovipositeur de l'insecte peut atteindre. En une année, B. psenes peut présenter trois générations se développant dans les caprifiguiers. Il est en mesure de polliniser des variétés femelles à chaque fois qu'il pénètre dans les figues. Les fruits fécondés renferment des graines gênant la consommation. De ce fait, les variétés parthénocarpiques sans graines sont les plus appréciées.

Pour leur commercialisation, les figuiers sont divisés en trois groupes : les variétés unifères, bifères et intermédiaires. Les figuiers unifères ne donnent que des figues d'automne, alors que les bifères fructifient une première fois au printemps (figuesfleurs) sur les rameaux formés l'année précédente, puis une seconde fois en été et en automne (figues d'automne) sur les pousses de l'année.

En Israël, six espèces de figuier ont été identifiées qui se différencient par leur type de fruit et leurs modalités de croissance et de fructification [1, 5] : Ficus carioca sylvestres, smirniaca, hortensis, intermedia, relicta et erinosis. Les variétés cultivées sont soit femelles, soit hermaphrodites. Elles appartiennent toutes aux types smirniaca (Smyrne), hortensis et intermedia (San Pedro).

\subsection{Croissance et fructification du figuier}

Le rameau du figuier est formé de deux types de tronçon [5-7] :

- le tronçon préformé existant sous forme d'ébauches dans le bourgeon initial, et constitué de 7 à 10 entrenœuds qui se

- le tronçon néoformé, nouveau-né sur le tronçon préformé, de 6 à 20 nœuds en général, qui continue la croissance.

Une présentation schématique (figure 1) a été proposée Lauri en 1995 [8].

Les figues-fleurs, qui peuvent être présentes sur des rameaux de différentes longueurs, sont surtout observées (63\%) sur les rameaux courts préformés de 6 à 10 entrenœuds [8]. Les figues d'automne se forment préférentiellement sur la partie néoformée $[5,7]$. Pour obtenir davantage de figuesfleurs, plusieurs techniques peuvent être préconisées : diminution de la fertirrigation déploient au débourrement ;
Figure 1.

Représentation schématique de la croissance annuelle d'un rameau de figuier (selon Lauri [8]). 
permettant de freiner la pousse des rameaux, pincement des rameaux longs en été favorisant la ramification et l'obtention de rameaux plus courts, ou bien chute artificielle des figues d'automne à un stade très jeune à l'aide de traitements chimiques [5, 7, 9-12].

\section{Matériel et méthodes}

Depuis l'Antiquité, des variétés de figuier très réputées ont été cultivées en Israël. D'autres citées dans d'anciens manuscrits - figuiers d'Égypte ou d'Iran, à figues jaunes ou des figues noires - ont été introduits au cours des temps. Cependant, peu de descriptions détaillées de leurs fruits sont disponibles [1].

Aujourd'hui, une lange gamme de variétés locales est cultivée, alors qu'il y a peu de cultivars introduits de l'étranger. Ces variétés semblent avoir été réimportées en Isräl, y a quelques centaines d'années [1]. Nous avons essayé de les identifier et de les répertorier.

Le nom des variétés locales exploitées en Israël, que nous avons repris dans nos travaux de caractérisation, est en langue arabe. Il évoque le site où la plante a été trouvée pour la première fois, un caractère principal du fruit comme sa couleur, son époque de maturité, les dimensions du fruit, etc. La détermination et la classification des différentes variétés collectées se sont révélées compliquées pour certaines raisons :

- cultivars changeant d'aspect selon les techniques de culture et le climat ;

- confusion dans le nom des variétés locales, une même variété pouvant porter plusieurs noms.

L'étude réalisée a porté sur la collecte de plusieurs centaines de clones et sur la production de vergers expérimentaux résultant principalement de quatre parcelles de cultures successives mises en place sur la station de recherches de Kefar Hanania, Haute Galilée, Israël, et sur l'observation de figues collectées dans l'ensemble du pays (Havat Matitiahou, haute montagne, et Névé Yaar, vallée d’Izreel).
Le verger expérimental est issu d'un premier verger planté avant 1948 avec des variétés ramassées par des agents de vulgarisation qui ont laissé très peu de données. Cette première parcelle ayant été ravagée par le feu, elle a été renouvelée et agrandie à partir d'une collecte et d'un tri plus ponctuel parmi des variétés présentes dans toutes les régions d'Israël. Ce verger a donc été composé des meilleures sélections trouvées auxquelles se sont ajoutées cinq variétés particulières retenues parmi 28 variétés importées de France (Pr. Rivals, Toulouse). Cette replantation a été renouvelée deux fois à cause de problèmes dus au sol (couches imperméables au sous-sol). La dernière parcelle mise en place qui fait l'objet de nos observations actuelles est composée de 81 variétés sélectionnées : 27 d'entre elles correspondent aux meilleures variétés trouvées dans les vergers précédents (récupérées à partir des arbres originaux), 19 trouvées dans le village de Kefar Tabor où se trouvait une ancienne collection mise en place à l'occasion d'un précédent travail, et un reliquat provenant des ressources génétiques conservées dans le cadre d'un projet national de «Création d'une banque génétique des espèces fruitières en Israël »[13].

En complément de l'étude des arbres de ce verger expérimental, des clones provenant de toutes les régions du pays ont été collectés, multipliés et systématiquement évalués. Ce travail de prospection s'est déroulé sur 5 ans ; il a été effectué par deux équipes qui ont parcouru, chaque année, les vergers et les bosquets de figuiers du territoire d'Israël, Gaza, Sinaï et le Golan pour récupérer tous les clones importants et surtout les spécimens anciens avec, de préférence, ceux cités dans la Bible et les anciennes écritures [13] qui présentent un intérêt historique, commercial et culturel. La multiplication des variétés retenues a été effectuée à l'aide de boutures à talon présentant une pousse de l'année et avec un morceau de bois de 2 ans prélevé à la base de l'arbre. Les greffons ont été désinfectés avant d'être traités en pépinière.

Cette collecte se poursuit actuellement partiellement. Certains arbres de la collection de figuiers qui a été ainsi constituée 
proviennent d'une prospection effectuée dans la région de Lachish, au sud d'Israël.

Tout le verger a été installé à Kefar Hanania à $400 \mathrm{~m}$ d'altitude, sur des terrasses anciennes ayant un sol de $50 \mathrm{~cm}$ de profondeur, "terra rossa » très caillouteuse (80 \% de pierres, donc environ $20 \mathrm{~cm}$ de terre nette).

Chaque variété retenue a été plantée, au hasard, dans deux blocs à raison de deux répétitions de quatre arbres par variété, plantés à une distance de plantation de $3 \mathrm{~m} \times 4 \mathrm{~m}$. L'irrigation et la fertilisation ont été assurées par des goutteurs autorégulés ayant un débit de $4 \mathrm{l} / \mathrm{h}$ et placés tous les mètres sur une seule rampe par rangée. Cependant, pour des raisons techniques de manque de pression et d'eau, dans les faits, les arbres ont reçu moins d'eau en été et sont donc restés toujours faibles. Ces problèmes ont été d'autant plus accentués que le sol de ce verger expérimental retenait très peu l'eau. Un tel substrat requiert habituellement une irrigation journalière intermittente qui donne d'excellents résultats dans d'autres stations de recherches ayant le même type de sol. Deux réplications de ce verger - l'un à Havat Matitiahou (haute montagne), l'autre à Névé Yaar (vallée d'Izreel) -, menées en très bonnes conditions de culture, ont permis de compléter alors nos résultats.

Le figuier est résistant à de basses températures $\left(-8{ }^{\circ} \mathrm{C}[1]\right)$, c'est la raison principale de sa grande dispersion jusque dans les plus hautes montagnes de notre pays. La plupart des variétés proviennent d'arbres très vieux (plusieurs centaines d'années) abandonnés, non irrigués, non soignés et donc difficiles à multiplier.

Notre étude a cherché à classer les figuiers et leurs clones en groupes différents à partir de clés fondamentales (figure 2) qui s'appuient sur la qualité des fruits étudiée sur la récolte de plusieurs années. Les arbres les plus intéressants de chaque groupe, considérés comme les meilleurs et donc recommandés aujourd'hui pour une culture moderne et intensive du figuier en Israël, sont présentés dans ce document.

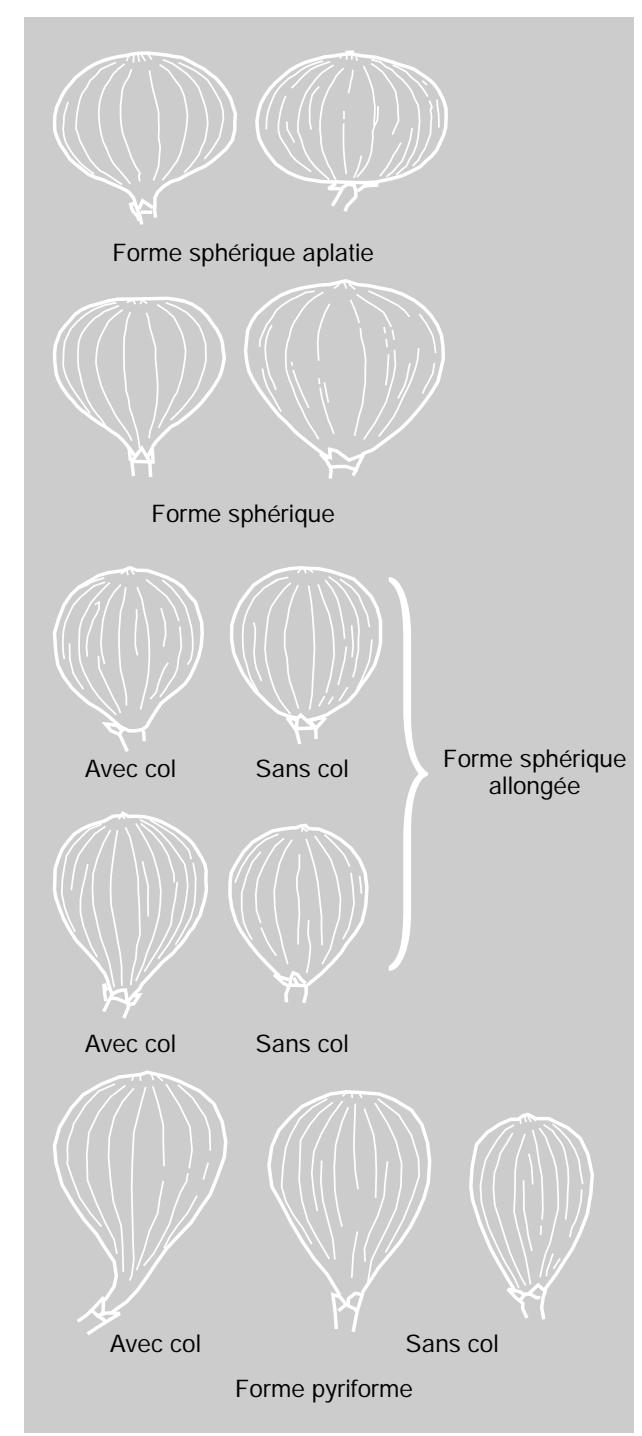

Figure 2.

Détermination des différentes formes de la figue (selon Goor [1]).
Un type particulier de figuier a été appelé «variété »lorsqu'il s'est révélé produire de mêmes figues dans plusieurs régions et sous des climats différents. Une variété présente ainsi des fruits ayant des caractères de base identiques qui vont être décrits. Le "clone » correspond à une variation des caractères de base de la variété.

\section{Résultats et classification des variétés étudiées}

La classification des variétés et des clones de figuiers qui a été adoptée repose tout 
Tableau I.

Caractères du fruit utilisés comme critères de base pour la classification des variétés et des clones de figuiers en Israël.

\section{Caractère considéré $\quad$ Modalités du caractère}

Couleur de la peau du fruit vert-jaune, vert-violet, brune, noire

Couleur de la chair Miel, rose, rougeâtre à rouge foncé

Forme du fruit

Pomme, sphérique arrondie, aplatie ou allongée, pyrimidique, figue (intermédiaire) avec ou sans col ${ }^{1}$

1 Voir figure 2 résultats sous climat de montagne froid et pluvieux.

Bosnia serait une variété introduite de Bosnie depuis longtemps; elle ressemble au type de figuier connu dans ce pays. Elle est aussi dénommée Bosnie, type SanPedro. La variété Nazareth décrite plus loin pourrait être un de ses mutants, le meilleur. Bosnia a un rendement faible, mais son fruit est de gros calibre et en partie éclaté ; il a un très bon goût. Cette variété est déconseillée pour la grande culture.

La variété Bukrati à gros fruits aplatis est très répandue dans toutes les régions d'Israël et réputée pour sa bonne qualité. Il en existe des clones particulièrement intéressants dont la culture peut être encouragée. Cependant, cette variété a l'inconvénient majeur d'avoir un ostiole ouvert qui favorise l'entrée des insectes nuisibles et le développement de moisissures.

Biyadi (= blanche) est une variété bien connue et appréciée, observée sous tous climats. Plusieurs clones en sont issus (Biyadi Al-Bire, Biyadi Ghazza, Biyadi Beit Jann, Levana Orot, etc.) parmi lesquels le meilleur est Biyadi Al-Bire qui produit un gros fruit à chair rouge-foncé, possédant le goût particulier très agréable (acidulé et sucré) de la variété.

La variété Dafur Ramallah (=figues-fleurs de Ramallah) ne donne pas de figues-fleurs, sauf en très bonnes conditions de culture, cependant sa maturité qui intervient au début de l'été est très précoce. Son fruit en général éclaté ne convient pas pour le commerce. À noter que l'arbre original trouvé dans les montagnes de Judée à Ramallah a de beaux fruits parfumés sans défauts, de même il donne de bons résultats à NéwéYa'ar où il bénéficie de très bonnes conditions de culture. La production de ce figuier serait donc influencée par son environnement climatique.

La variété Khadari (= verte, également dénommée A'khadari) est décrite différemment par plusieurs auteurs. Le plus important de ses clones a été collecté à Deir Hanna. Son fruit, sans grande spécificité, a une taille moyenne. 
Table II.

Classement des variétés de figuier appartenant au groupe des fruits vert-jaune, à partir des caractéristiques de leurs fruits.

\begin{tabular}{|c|c|c|c|c|c|c|c|c|c|c|c|c|}
\hline \multirow[t]{2}{*}{$\mathrm{N}^{0}$} & \multirow[t]{2}{*}{ Variété } & \multirow{2}{*}{$\begin{array}{c}\text { Date } \\
\text { de maturité }\end{array}$} & \multirow[t]{2}{*}{ Pédoncule } & \multirow{2}{*}{$\begin{array}{l}\text { Calibre } \\
\text { du fruit }\end{array}$} & \multirow[t]{2}{*}{ Goût ${ }^{1}$} & \multirow[t]{2}{*}{ Couleur $^{1}$} & \multirow[t]{2}{*}{ Apparence $^{1}$} & \multicolumn{2}{|c|}{ Épiderme } & \multirow{2}{*}{$\begin{array}{l}\text { Couleur } \\
\text { de la chair }\end{array}$} & \multirow[t]{2}{*}{ Graines } & \multirow[t]{2}{*}{ Forme du fruit } \\
\hline & & & & & & & & Couleur & Épaisseur & & & \\
\hline & Excel & Mi-saison & Long & Moyen & 4,2 & 4,4 & 4,1 & $\begin{array}{l}\text { Vert jaune + } \\
\text { lenticelles }\end{array}$ & Fin & Rose brun & \pm & $\begin{array}{l}\text { Turbinée + } \\
\text { col à la base }\end{array}$ \\
\hline 2 & Bosnia & Mi-saison & Court & Gros & 5,0 & 4,8 & 4,8 & Vert clair + veinée & Moyen gros & Rose miel & + & $\begin{array}{l}\text { Turbinée aplatie } \\
\text { + col à la base }\end{array}$ \\
\hline 3 & Bukrati & Mi-saison & Court & Moyen gros & 4,4 & 4,4 & 4,8 & Vert clair + veines & Fin & Rouge & \pm & Turbinée + col à la base \\
\hline 4 & Biyadi & Mi-saison & Court & Moyen & 4,0 & 4,6 & 4,4 & Vert + lenticelles & Moyen & Rouge & \pm & Turbinée aplatie \\
\hline 5 & Dafur Ramallah & Précoce & Moyen & Moyen & 4,3 & 4,8 & 4,7 & Vert + lenticelles & Fin moyen & Rose & \pm & Turbinée aplatie \\
\hline 6 & Khadari & Mi-saison & Moyen & Moyen petit & 4,8 & 4,5 & 4,5 & Vert clair + veines & Gros & Rose & \pm & Sphérique \\
\hline 7 & Hamari Wadi Salame & Mi-saison & Moyen & Petit & 4,6 & 4,5 & 4,5 & Vert & Fin moyen & Rouge foncé & + & Turbinée \\
\hline 8 & Tu'ali & Mi-saison & Long & Petit & 3,7 & 5,0 & 3,1 & Vert jaune & Fin moyen & Rose foncé & - & Pyriforme \\
\hline 9 & Te'ena Yeruqqa & Mi-saison & Fin & Moyen petit & 3,0 & 4,0 & 3,0 & Vert & Moyen & Rose & \pm & Sphérique aplatie \\
\hline 10 & Kabari & Mi-saison & Moyen & Moyen & 5,0 & 4,6 & 4,6 & Vert + veines & Moyen & Rose miel & - & Pyriforme \\
\hline 11 & Kabari Kafr Hares & Mi-saison & Moyen & Gros & 5,0 & 5,0 & 5,0 & Vert clair + veines & Gros & Rose miel & - & Aplatie + col à la base \\
\hline 12 & Khiyali & Mi-tardive & Moyen & Petit & 3,9 & 4,3 & 4,1 & Vert jaune & Moyen & Rose foncé & - & Sphérique aplatie \\
\hline 13 & Nazareth & Mi-précoce & Fin long & Gros & 4,9 & 4,8 & 4,7 & Vert clair + veines & Moyen gros & Rose miel & \pm & Turbinée \\
\hline 14 & Suchard & Mi-saison & Moyen & Moyen & 5,0 & 5,0 & 4,5 & Vert clair + veines & Gros & Rose miel & - & Turbinée + col à la base \\
\hline 15 & Smyrna & Précoce & Court & Très gros & 4,6 & 4,9 & 4,5 & Vert clair- jaune & Gros & Rose miel & + & Sphérique \\
\hline 16 & Safari & Tardive & Moyen & Gros & 4,0 & 5,0 & 4,0 & Vert jaune & Moyen & Rose & - & Aplatie \\
\hline 17 & Ajluni & Très tardive & Fin moyen & Petit & 4,6 & 4,5 & 4,0 & Vert + lenticelles & Moyen & Rouge & + & Pyriforme \\
\hline 18 & Adasi & Mi-saison & Fin & Petit & 3,4 & 2,5 & 3,0 & Vert-brun + lenticelles & Fin & Rose rouge & \pm & Turbinée aplatie \\
\hline 19 & Porat Yeruqqa & Mi-saison & Moyen & Petit & 3,8 & 3,8 & 3,5 & Vert clair & Fin & Blanc, rouge & \pm & Turbinée \\
\hline 20 & Frum & Précoce & Court & Moyen & 4,3 & 4,7 & 4,5 & $\begin{array}{l}\text { Vert + veines } \\
+ \text { lenticelles }\end{array}$ & Moyen & Rose miel & \pm & Turbinée \\
\hline 21 & Kadota & Mi-saison & Gros moyen & Gros & 4,8 & 4,5 & 3,9 & Vert + lenticelles & Moyen & Rose foncé & \pm & Turbinée \\
\hline 22 & Col de Dame Blanche & Mi-saison & Petit & Très gros & 4,2 & 4,7 & 4,7 & $\begin{aligned} & \text { Vert clair } \\
+ & \text { lenticelles }\end{aligned}$ & Moyen gros & Rose rouge & \pm & Turbinée \\
\hline 23 & Conadria & Mi-saison & Large court & Moyen petit & 4,4 & 4,8 & 4,3 & Vert & Moyen & Rouge miel & - & Turbinée aplatie \\
\hline 24 & Shahami & Précoce & Court & Petit & 4,5 & 4,8 & 4,0 & Vert clair + lenticelles & Moyen & Rose rouge & \pm & Turbinée \\
\hline 25 & Shatawi & Tardive & Fin court & Petit & 4,0 & 3,9 & 3,9 & Vert clair & Moyen fin & Rose rouge foncé & é - & Turbinée + col à la base \\
\hline 26 & Tena & Mi-saison & Court & Moyen & 5,0 & 4,4 & 4,0 & Vert clair + veines & Moyen gros & Rose miel & - & Turbinée + col à la base \\
\hline 27 & $\mathrm{~K}-4$ & Précoce & Court & Petit & 4,5 & 4,5 & 3,8 & Vert jaune clair & Moyen fin & Rose & + & Turbinée aplatie \\
\hline
\end{tabular}

${ }^{1}$ Les caractéristiques de goût, couleur et apparence sont exprimées par une note qualitative variant de 1 (modalité la moins intéressante) à 5 (modalité la plus intéressante). 
Le clone Hamari Wadi Salame (= variété rouge) est le plus intéressant de la variété Hamari. En fin de maturité, son fruit est rougeâtre. Habituellement, il est vert légèrement violet, doux, de bonne qualité avec une chair rouge foncé et un épiderme épais qui lui permet de sécher sur l'arbre. Cette figue sans graines, très appréciée, est cueillie déshydratée et vendue en «fruits sucrés »à goût de miel parfumé, de très grande qualité.

La variété Tu'ali (= la longue) a un fruit très allongé. Selon les auteurs, elle a différents noms et descriptions. Elle donne une belle et grosse figue vert-jaune de très bonne qualité et de belle présentation. Elle exige de bonnes conditions de culture, d'irrigation et de fertilisation.

Te'ena Yeruqqa (= figue verte) est un cultivar ressemblant à Khadari mais l'aspect de son fruit, de qualité moyenne, en est différent.

La variété Kabari (=la grande), sans intérêt spécial, ne produit de gros fruits que lorsqu'elle est cultivée dans de bonnes conditions. Le cultivar Kabari Kafr Hares, qui a été collecté dans le village de Harès, à gros et beau fruit, serait le meilleur clone de cette variété, mais l'arbre n'est pas très productif.

Khiyali (= fruit beau comme le cheval arabe) a un petit fruit éclaté et ne présente pas d'intérêt particulier.

Le cultivar vert-jaune de Nazareth (trouvée dans un couvent à Nazareth) est le figuier le plus cultivé et le plus répandu en Israël ; il est présent dans tout le Bassin méditerranéen sous différents noms. Plusieurs clones en sont issus qui ont été identifiés en fonction de la date de maturité et de la qualité des fruits. En été, Nazareth est la variété la plus précoce. L'un de ses clones a la particularité de produire régulièrement de bonnes récoltes de figues-fleurs (fruits pesant de 70 à $120 \mathrm{~g}$, avec un diamètre de 50 à $60 \mathrm{~mm}$ et un goût mielleux) et la plupart des clones se ressemblent dans la forme du fruit, la croissance et la vigueur des arbres. Le cultivar Nazareth lui-même a une très grande végétation. La récolte porte sur deux vagues de production : en général, en Israël, les figues-fleurs sont cueillies en mai et les figues d'été en juillet. Dans la vallée chaude du Jourdain, les deux vagues se récoltent un mois avant. Les fruits d'été, souvent de type femelle sur arbres adultes ou de type parthénocarpique sur arbres jeunes, avec ou sans fécondation, sont jaune-doré, de très bonne qualité et de belle présentation. Ils ont une chair mielleuse et parfumée ; ils résistent bien aux manipulations du triage et du transport, ont une bonne tenue sur les étalages des magasins et donnent aussi des figues sèches de très bonnes qualités.

Parmi les nombreux clones du cultivar Nazareth échantillonnés, les plus intéressants gardés en observation sont Yafo Kadoori (différent de la variété Qifoni connue par les Arabes), Hanni'el Yeruqqa, Kurt, Shahamani (différent de la variété Shuhmani), Kedana'it, Biyadi Beih Jann (à ne pas confondre avec la variété Biyadi qui a une chair rouge), Asisi, Gevar'am, Qubeiba Ma'arav Yeruqqa, Nir Aqiva, Bahari Yeruqqa, Qomémiyyut, Kefar Uriyya, Zor'a et Qubeiba Mizrah. Parmi eux, les clones Yafo Kadoori, Kurt, Shahamani et Kefar Uriyya sont les meilleurs pour la production commerciale de figues d'été.

À l'issue de nos travaux sur la production de figues-fleurs, le clone Nazareth du type San-Pedro serait, jusqu'à présent, celui qui produirait les plus beaux et gros fruits, et une récolte annuelle stable et importante.

Suchard est une variété introduite à partir de la collection «Rivals » de Toulouse (France). Elle ressemble beaucoup à Nazareth, mais ne produit pas de figues-fleurs. Son fruit est de très gros calibre et possède des qualités organoleptiques excellentes.

Smyrna est un cultivar très ancien, réintroduit de Turquie et très répandu en Israël. Il donne une excellente grosse figue utilisée pour la consommation en fruit frais, pour l'exportation ou pour la production de fruits séchés. En Turquie, où elle porte des noms différents, c'est la principale variété cultivée.

Safari (= figue jaune) est une variété très répandue en Israël. Elle est tardive et la qualité de ses fruits est moyenne, bien 
qu'elle puisse être améliorée par de bonnes conditions de culture en terres profondes.

La variété Ajluni, qui provient de la ville d'Ajlun en Jordanie, est très tardive ; elle a de petits fruits dont les qualités organoleptiques et la présentation sont moyennes. Elle pourrait donner de meilleurs résultats avec l'utilisation de techniques culturales plus performantes et une lutte plus efficace contre les insectes et les acariens qui l'endommagent fortement en fin de saison.

Le cultivar Adasi (= lentille) produit de petits fruits tardifs et ne présente pas d'intérêt particulier. De même, la variété verte Porat Yeruqqa ne mérite pas d'attention spéciale.

Le cultivar Frum, récupéré chez M. Frum en Judée, est un clone de Bosnya qui ressemble à la variété Nazareth, mais il en est différent par certains caractères. Il ne présente pas d'intérêt particulier.

La variété Kadota (= Doutato) a été introduite en Israël à partir d'Italie depuis longtemps. Le clone étudié porte des fruits d'été plus petits et différents de ce qui peut être observé, en bonnes conditions de culture, en Europe et en Californie où c'est le type de figuier le plus répandu. Il peut donner d'excellents résultats lorsqu'il est conduit avec des tailles bien étudiées en hiver et des techniques de culture particulières permettant une récolte groupée. Il donne un fruit frais, mielleux, de grande qualité et des figues sèches excellentes. Le clone original, qui a été décrit par Goor [1], est le meilleur ; il se rencontre dans plusieurs vergers de la plaine Izreel.

La variété «Col de Dame Blanche »provient de la collection Rivals (Toulouse, France). En France, comme en Isräl, elle donne un très gros et beau fruit, de bonne qualité. Elle est recommandée en montagne où il fait plus froid l'hiver et plus frais l'été. Dans de bonnes conditions, elle donne de bonnes récoltes en début août à destination du marché local ou de l'exportation.

La variété Conadria également issue de la collection Rivals a des performances moyennes qui ne justifient pas de la recommander en Israël.
Shahamani (= huileuse) est un cultivar très précoce en maturité, qui a été collecté à Deir Hanna. Pour l'ensemble de ses principaux caractères, il ressemble à Biyadi, dont il est un clone.

Shatawi (= hivernale) est une variété tardive qui mûrit en automne. Parmi tous les cultivars portant ce nom, certains sont plus intéressants que d'autres pour la production des figues tardives. En général, le fruit est assez petit, de qualité moyenne et difficile à commercialiser.

Tena (= figue en hébreu) est un figuier importé du Texas. Ses caractéristiques ressemblent à celles de la variété Nazareth, mais il ne produit pas de figues-fleurs.

K-4 (= Mdu-A-4) est une sélection du verger expérimental de Kefar Hanania qui donne de très bonnes récoltes. Son petit fruit qui sèche sur l'arbre est très doux, sans graines et de très bonne qualité. Il est consommé en apéritif comme fruit spécial déshydraté.

\subsection{Deuxième groupe : figuiers à fruits vert-violet}

De même que pour les variétés des figuiers du premier groupe, la description des figuiers à fruits vert-violet présentée sur le tableau III peut être complétées par quelques précisions.

Adom Gadol (= gros fruit rougeâtre) est une des meilleures variétés commerciales recommandées pour ce groupe de figues. Le fruit est de très gros calibre, a de très bonnes qualités gustatives, une belle présentation et il ne souffre pas des manipulations du triage et du transport.

Zaraqi Kadoori (= couleur bleue) est un clone collecté à Kadoori, dans la vallée intérieure d'Izreel où il est très répandu. L'arbre a un port ouvert très étalé. Le fruit est bon et ressemble à celui de la variété Mission. Le clone Hanni'el Shahora que nous avons étudié est un mutant de Zaraqi ; il a un fruit de qualité et de calibre moyens.

Le cultivar Hamari BeitJann (=rougeâtre) est semblable à la variété Hamari qui se trouve dans les vergers arabes. Le fruit de 
Tableau III.

Classement des variétés de figuier appartenant au groupe des fruits vert-violet, à partir des caractéristiques de leurs fruits.

\begin{tabular}{|c|c|c|c|c|c|c|c|c|c|c|c|c|}
\hline \multirow{2}{*}{\multicolumn{2}{|c|}{$N^{\circ}$ Variété }} & \multirow{2}{*}{$\begin{array}{c}\text { Date } \\
\text { de maturité }\end{array}$} & \multirow[t]{2}{*}{ Pédoncule } & \multirow{2}{*}{$\begin{array}{l}\text { Calibre } \\
\text { du fruit }\end{array}$} & \multirow[t]{2}{*}{ Goût ${ }^{1}$} & \multirow[t]{2}{*}{ Couleur $^{1}$} & \multirow[t]{2}{*}{ Apparence $^{1}$} & \multicolumn{2}{|l|}{ Épiderme } & \multirow{2}{*}{$\begin{array}{l}\text { Couleur } \\
\text { de la chair }\end{array}$} & \multirow[t]{2}{*}{ Graines } & \multirow[t]{2}{*}{ Forme du fruit } \\
\hline & & & & & & & & Couleur & Épaisseur & & & \\
\hline 1 & Gadol Adom & Mi-tardive & Gros moyen & Gros & 4,3 & 5,0 & 5,0 & $\begin{array}{l}\text { Vert violet strillé } \\
+ \text { lenticelles }\end{array}$ & Moyen & Rouge rose & - & $\begin{array}{l}\text { Turbinée aplatie } \\
+ \text { col à la base }\end{array}$ \\
\hline 2 & Zraqi Kadoori & Mi-saison & Moyen & Petit & 3,9 & 3,6 & 3,7 & Vert violet + lenticelles & Moyen & Rose foncé & - & Turbinée \\
\hline 3 & Hamari Beit Jann & Mi-saison & Gros court & Moyen gros & 4,6 & 4,5 & 4,5 & Vert violet + lenticelles & Gros & Rouge & \pm & Turbinée \\
\hline 4 & Hamudi & Mi-tardive & Moyen & Très gros & 3,9 & 4,8 & 5,0 & Vert violet veiné + lenticelles & Gros & Rouge foncé & \pm & Turbinée aplatie \\
\hline 5 & Tu'ali & Tardive & Long & Moyen petitt & 3,8 & 4,4 & 3,8 & Vert violet brun & Fin & Rose foncé & - & Pyriforme \\
\hline 6 & $\mathrm{~K}-16$ & Mi-tardive & Moyen & Moyen & 3,7 & 4,5 & 5,0 & Violet jaune & Fin & Rose foncé & - & Aplatie \\
\hline 7 & Mu'azi & Tardive & Moyen & Petit & 4,6 & 4,0 & 3,8 & $\begin{array}{c}\text { Vert violet } \\
+ \text { veines violettes }\end{array}$ & Moyen & Miel & \pm & Turbinée \\
\hline 8 & Mission & Mi-saison & Moyen & Petit & 3,9 & 4,3 & 4,1 & Vert violet + lenticelles & Moyen & Rose foncé & - & Turbinée \\
\hline 9 & Saba'i & Tardive & Court & Moyen & 4,9 & 4,8 & 4,9 & Vert violet jaune & Moyen gros & Rose & - & Turbinée aplatie \\
\hline 10 & Asali & Tardive & Moyen long & Moyen & 4,6 & 4,8 & 4,2 & $\begin{array}{c}\text { Vert violet } \\
+ \text { veines violettes }\end{array}$ & Moyen & Miel & - & Aplatie \\
\hline 11 & Flanders & Mi-tardive & Long fin & Moyen petit & 4,6 & 3,7 & 4,4 & $\begin{array}{l}\text { Vert violet + veines } \\
\text { violettes + lenticelles }\end{array}$ & Moyen & Rose foncé & \pm & Pyriforme \\
\hline 12 & Cope & Mi-saison & Moyen large & Gros & 3,5 & 5,0 & 4,8 & Vert violet + lenticelles & Moyen & Rouge rose & - & Aplatie \\
\hline 13 & Karak'i & Tardive & Fin & Petit & 3,8 & 3,0 & 3,2 & Vert violet + lenticelles & Moyen & Rose miel & - & Turbinée aplatie \\
\hline 14 & $P-20$ & Mi-saison & Très court & Moyen petit & 4,6 & 4,5 & 4,8 & Violet foncé + lenticelles & Moyen & Rose rouge foncé & $\dot{e} \pm$ & Turbinée + col à la base \\
\hline
\end{tabular}

Les caractéristiques de goût, couleur et apparence sont exprimées par une note qualiative variant de 1 (modalité la moins intéressante) à 5 (modalité la plus intéressante).

\section{Tableau IV.}

Classement des variétés de figuier appartenant au groupe des fruits bruns, à partir des caractéristiques de leurs fruits.

\begin{tabular}{|c|c|c|c|c|c|c|c|c|c|c|c|c|}
\hline \multirow{2}{*}{\multicolumn{2}{|c|}{$\mathrm{N}^{\circ}$ Variété }} & \multirow{2}{*}{$\begin{array}{c}\text { Date } \\
\text { de maturité }\end{array}$} & \multirow[t]{2}{*}{ Pédoncule } & \multirow{2}{*}{$\begin{array}{l}\text { Calibre } \\
\text { du fruit }\end{array}$} & \multirow[t]{2}{*}{ Goût ${ }^{1}$} & \multirow[t]{2}{*}{ Couleur $^{1}$} & \multirow[t]{2}{*}{ Apparence ${ }^{1}$} & \multicolumn{2}{|c|}{ Épiderme } & \multirow{2}{*}{$\begin{array}{l}\text { Couleur } \\
\text { de la chair }\end{array}$} & \multirow[t]{2}{*}{ Graines } & \multirow[t]{2}{*}{ Forme du fruit } \\
\hline & & & & & & & & Couleur & Épaisseur & & & \\
\hline 1 & Ghazali & Tardive & Moyen & Gros & 4,3 & 4,5 & 4,7 & Brun violet & Moyen & Rouge & - & Sphérique aplatie \\
\hline 2 & Khurtamani & Mi-tardive & Long fin & Gros & 4,0 & 4,5 & 4,5 & Brun & Gros & Brun & \pm & Turbinée pyriforme \\
\hline 3 & Hamadi & Mi-saison & Court & Moyen & 4,0 & 4,5 & 5,0 & Brun violet & Moyen & Rouge & + & Sphérlque \\
\hline
\end{tabular}


qualité médiocre est toujours éclaté, phénomène observé dans plusieurs régions où sont entretenues nos parcelles d'études.

Le fruit de la variété Hamudi (=jolie) est gros et beau, avec un goût peu sucré. Ce figuier est apprécié des producteurs qui le trouvent facile à cultiver et à vendre. Il est recommandé pour le commerce des variétés de la mi-saison tardive.

Tu'ali Shehora (= longue noire) est une variété présentant des performances peu stables dans les régions chaudes où elle donne parfois de petits fruits sans grande qualité. Cependant, chez les arbres jeunes, sa production peut être plus intéressante. Avec de meilleures techniques culturales, ce figuier peut être planté en haute montagne.

Le clone 6-K-16 provient de l'ancienne collection de Kefar Hanania. Son fruit est très gros, beau et d'excellente qualité. C'est une variété recommandée pour la commercialisation.

Le clone Mu'azi (= allongée comme une banane) a été collecté à Gaza, Beit Lahiya. Il est diffèrent de celui du même nom, connu en Israël où il est également appelé Asali ou Khurtamani. Il donne un petit fruit peu intéressant.

La variété Mission, à gros et beau fruit noir, a été importée plusieurs fois à partir de différents pays dont l'Espagne et la Grèce. C'est la «Negra espagnole », clone qui produit des figues plutôt vert-violet, à forme pyramidale. Les fruits des variétés Adler et Hanni'el sont identiques à ceux de la variété Mission mais l'arbre a un port différent.

Saba'i (= sept mois) est une variété très connue en Israël ; elle est tardive en montagne comme à Kefar Hanania. Le fruit est gros et excellent.

La variété Asali (= mielleux) a été collectée à Deir Hanna, en Basse Galilée. Le clone qui a été étudié est moins intéressant que celui qui a été décrit par d'autres auteurs. Il a un fruit très tardif, de qualité moyenne, en partie éclaté. Il semble pouvoir s'adapter en haute montagne.
La variété Flanders, en provenance du Texas a un fruit qui devient brun à maturité avancée ; sa présentation et sa qualité sont faibles.

Cope (= la figue d'Eva) est une variété intéressante qui a des feuilles géantes avec peu de lobes. Son fruit est beau, gros, succulent, identique à A-3.

Karak'i (= couleur de tortue) est un cultivar à petit fruit tardif, qui ne présente pas un intérêt majeur.

La variété P-20, sélectionnée dans le verger expérimental de Kefar Hanania, produit une bonne figue noire dont la belle couleur se développe à complète maturité seulement. Son fruit, de calibre moyen en plaine et de gros calibre en haute montagne, est de très bonne qualité. Cette variété est prometteuse.

\subsection{Troisième groupe : figuiers à fruits brun strié}

Ce groupe ne comporte que trois variétés (tableau IV).

Le clone Ghazali (= belle figue ressemblant à une gazelle) est différent de la variété Ghazali de Beit Jann ; il a un fruit gros, beau et tardif.

Khurtamani (= long bec) est une variété très connue et répandue dans les vergers arabes. Elle donne de belles figues, grosses, sucrées ; son goût est moins mielleux que la plupart des autres figues.

Le clone Hamadi (= acidulé) produit un fruit qui ressemble à celui de la variété Khurtamani, mais il est de moins bonne qualité. Par ailleurs, sa forme évoque celle d'une pomme.

\subsection{Quatrième groupe : figuiers à fruits noirs}

Les principales caractéristiques des figuiers de ce groupe (tableau V) peuvent être complétées par les quelques informations qui suivent.

La variété Adriatica est une introduction ancienne provenant de la Côte adriatique et 
Tableau V.

Classement des variétés de figuier appartenant au groupe des fruits noirs, à partir des caractéristiques de leurs fruits.

\begin{tabular}{|c|c|c|c|c|c|c|c|c|c|c|c|c|}
\hline \multirow[t]{2}{*}{$\mathrm{N}^{\circ}$} & \multirow[t]{2}{*}{ Variété } & \multirow{2}{*}{$\begin{array}{c}\text { Date } \\
\text { de maturité }\end{array}$} & \multirow[t]{2}{*}{ Pédoncule } & \multirow{2}{*}{$\begin{array}{l}\text { Calibre } \\
\text { du fruit }\end{array}$} & \multirow[t]{2}{*}{ Goût $^{1}$} & \multirow[t]{2}{*}{ Couleur $^{1}$} & \multirow[t]{2}{*}{ Apparence $^{1}$} & \multicolumn{2}{|c|}{ Épiderme } & \multirow{2}{*}{$\begin{array}{l}\text { Couleur } \\
\text { de la chair }\end{array}$} & \multirow[t]{2}{*}{ Graines } & \multirow[t]{2}{*}{ Forme du fruit } \\
\hline & & & & & & & & Couleur & Épaisseur & & & \\
\hline 1 & Adriatica & Mi-tardive & Court petit & Moyen petit & 2,9 & 4,5 & 4,4 & Lenticelles violettes & Fin & Rose foncé & + & Aplatie \\
\hline 2 & Italqit Shehora & Mi-saison & Petit fin & Petit & 4,5 & 4,7 & 4,8 & $\begin{array}{l}\text { Violet foncé noir } \\
+ \text { lenticelles }\end{array}$ & Moyen fin & Rose miel & - & Turbinée aplatie \\
\hline 3 & Diehl Noire & Mi-saison & Moyen & Moyen petit & 4,2 & 4,6 & 4,4 & $\begin{array}{l}\text { Violet noir } \\
+ \text { lenticelles }\end{array}$ & Moyen fin & Rouge & \pm & Turbinée aplatie \\
\hline 4 & Harubi & Mi-tardive & Court & Moyen petit & 3,7 & 4,6 & 4,5 & $\begin{array}{l}\text { Violet foncé } \\
+ \text { lenticelles }\end{array}$ & Moyen gros & Rose foncé & \pm & Turbinée aplatie \\
\hline 5 & Yoqne'am Shehora & Précoce & Court & Moyen & 3,8 & 4,6 & 4,6 & $\begin{array}{l}\text { Violet foncé noir } \\
+ \text { lenticelles }\end{array}$ & Moyen gros & Rose miel & \pm & Turbinée pyriforme \\
\hline 6 & Mat 1 & Tardive & Fin & Moyen petit & 3,8 & 4,8 & 4,0 & Violet foncé noir & Gros & Rose clair miel & \pm & Turbinée \\
\hline 7 & Su'adi & Tardive & Court fin & Petit & 4,1 & 4,3 & 3,3 & Noir & Moyen fin & Miel & + & Turbinée aplatie \\
\hline 8 & Col de Dame Noire & Mi-saison & Petit & Petit & 3,5 & 4,8 & 4,5 & Noir + lenticelles & Fin & Rouge & + & Turbinée \\
\hline 9 & Robine & Mi-saison & Court & Moyen petit & 4,7 & 4,7 & 4,7 & Noir & Moyen fin & Rose foncé miel & I - & Aplatie \\
\hline 10 & Shaltufat Al-Abed & Mi-saison & Long & Petit & 4,2 & 4,7 & 4,4 & Noir & Fin & Rose foncé & - & Aplatie \\
\hline
\end{tabular}

${ }^{1}$ Les caractéristiques de goût, couleur et apparence sont exprimées par une note qualiative variant de 1 (modalité la moins intéressante) à 5 (modalité la plus intéressante). 
qui ressemble à Harubi, décrit plus bas, dont elle a les mêmes caractères.

Italqit Shehora (=italienne noire) est une vieille introduction d'Italie, identique au clone Adler. Sa figue a une belle présentation, un bon goût et sa qualité est excellente. Certains clones avec fruits plus petits doivent être délaissés. La dernière variété réimportée d'Italie s'appelle Tuvia noire, du nom de la personne qui l'a réintroduite. Elle donne un arbre à végétation vigoureuse et feuillage dense ; de ce fait, son fruit, identique à la variété Italqit Shehora d'origine, est de bonne qualité en montagne. Cette variété peut être recommandée pour une plantation en grands vergers commerciaux.

Le clone Diehl Noire, identique à la variété Col de Dame Noire, provient de la collection Rivals (France). Il a besoin de bonnes conditions de culture pour donner de gros fruits de qualité.

La variété Harubi (= goût du caroubier) et ses clones se ressemblent. Ce figuier est répandu dans les vergers arabes et se distingue par son goût de caroubier. Les fruits récoltés dans le verger expérimental de Kefar Hanania sont acidulés, assez sucrés, de grosseur moyenne et très beaux. La variété est répandue dans les plaines intérieures et sur la côte d'Israël. Parmi les clones étudiés, ceux qui semblent les plus intéressants sont Mevo Horon, Porat Shehora, Shehora BeitJann, Harubi Ramallah, Bet Guvrin Shehora et Biyadi Ramallah (différent de Biyadi). Ces différents clones se distinguent par le port de l'arbre.

Yoqne'am Shehora, figuier à fruits noirs trouvé dans le village de Yoqne'am, dans les montagnes d'Efraim, est une variété peu productive, mais à beaux fruits. Elle ne peut être recommandée pour une culture commerciale.

Mat 1, une sélection de la station Kefar Hanania, est un cultivar qui donne un fruit de qualité et de calibre moyens sans intérêt particulier.

Su'adi ( = noire, également dénommée Smari) est une variété donnant des fruits de qualité médiocre.

La variété Col de Dame Noire provient de la collection Rivals. Elle est excellente à
Toulouse (France) mais, en Israël, les arbres ne se développent pas. Elle exige des hivers très froids, beaucoup d'eau et de très bonnes conditions de culture. Son fruit ressemble à celui de la variété Diehl noire, mais en plus gros.

La variété Robine, également importée de la collection Rivals, est très productive. Elle produit un beau fruit de grosseur moyenne, de bonne qualité, à la peau ferme et dure. Cela lui confère une bonne tenue en étalage et une bonne résistance aux manipulations de cueillette, triage et transport. De sévères tailles d'élagage favorisent l'obtention de figues à gros calibres.

La variété Shaltufat al-Abed (= les lèvres de l'esclave) a été collectée sur le bord d'une rivière en Haute Galilée. Elle donne un gros fruit, très beau, de bonne qualité. Elle exige de bonnes conditions de culture et d'irrigation.

\section{Croissance des arbres}

Les figuiers des variétés étudiées ont présenté de grandes variations dans la végétation des arbres, la longueur, la ramification des rameaux et leur intensité (tableau VI). Ces caractères dépendent des conditions de culture, de la qualité des tailles, de l'irrigation et de la fertilisation, mais ils sont surtout déterminés par la structure génétique de la variété ou du clone.

Chacune des variétés est ainsi caractérisée par un port principalement étalé, semi étalé ou dressé. Elles peuvent aussi se distinguer par leur feuille de dimensions différentes - parfois géante comme celle de la variété Cope, longue de $50 \mathrm{~cm}$ - avec ou sans lobes, avec ou sans dentelure, présentant une épaisseur ou une couleur différente (sombre à claire). L'ensemble de ces paramètres a permis de classer les variétés et clones étudiés (tableau VI), et donc d'identifier des caractéristiques communes à tous les clones d'une même variété. Ainsi, tous les clones de la variété Nazareth ont un port d'arbre très dressé et une feuille identique. 
Tableau VI.

Caractéristiques des différentes variétés de figuier cultivées en Israël (site expérimental de Kefar Hanania, Haute Galilée).

\begin{tabular}{|c|c|c|c|c|c|c|c|}
\hline $\mathrm{N}^{\circ}$ & Variété & Productivité & $\begin{array}{c}\text { Stabilité } \\
\text { des récoltes }\end{array}$ & $\begin{array}{c}\text { Quantité } \\
\text { de pousses }{ }^{1}\end{array}$ & $\begin{array}{l}\text { Longueur } \\
\text { de pousses }\end{array}$ & $\begin{array}{c}\text { Taille } \\
\text { des arbres }\end{array}$ & $\begin{array}{c}\text { Port } \\
\text { des arbres }\end{array}$ \\
\hline \multicolumn{8}{|c|}{ Groupe des figues vert-jaune } \\
\hline 1 & Excel & Moyenne & Stable & 4,5 & 4,8 & 4,8 & Étalé \\
\hline 2 & Bosnia & Grande & Stable & 4,8 & 4,8 & 4,8 & Érigé \\
\hline 3 & Bukrati & Moyenne & Stable & 4,0 & 4,0 & 4,5 & Moyen \\
\hline 4 & Biyadi & Moyenne & Stable & 4,2 & 4,2 & 4,3 & Érigé \\
\hline 5 & Dafur Ramallah & Faible & Stable & 3,5 & 3,5 & 3,5 & Moyen \\
\hline 6 & Khadari & Faible & Stable & 3,5 & 3,5 & 3,5 & Étalé \\
\hline 7 & Hamari Wadi Salame & Moyenne & Stable & 4,5 & 5,0 & 4,5 & Étalé \\
\hline 8 & Tu'ali & Moyenne & Stable & 4,5 & 4,5 & 4,8 & Érigé \\
\hline 9 & Te'ena Yeruqqa & Moyenne & Stable & 4,0 & 4,0 & 4,0 & Érigé \\
\hline 10 & Kabari & Faible & Stable & 4,0 & 4,0 & 4,5 & Érigé \\
\hline 11 & Kabari Kafr Hares & Faible & Stable & 4,0 & 4,5 & 4,0 & Érigé \\
\hline 12 & Khiyali & Faible & Stable & 3,5 & 3,5 & 3,5 & Étalé \\
\hline 13 & Nazareth & Moyenne & Stable & 4,1 & 4,0 & 4,2 & Érigé \\
\hline 14 & Suchard & Faible & Stable & 4,0 & 4,0 & 4,0 & Érigé \\
\hline 15 & Smyrna & Grande & Stable & 4,5 & 4,5 & 4,0 & Érigé \\
\hline 16 & Safari & Moyenne & Alternante & 3,0 & 3,0 & 3,5 & Moyen \\
\hline 17 & Ajluni & Moyenne & Stable & 3,5 & 3,5 & 3,5 & Étalé \\
\hline 18 & Adasi & Moyenne & Alternante & 4,0 & 4,3 & 4,0 & Étalé \\
\hline 19 & Porat Yeruqqa & Moyenne & Stable & 3,5 & 3,5 & 3,5 & Étalé \\
\hline 20 & Frum & Moyenne & Alternante & 4,5 & 5,0 & 4,5 & Érigé \\
\hline 21 & Kadota & Moyenne & Stable & 4,5 & 4,5 & 4,5 & Moyen \\
\hline 22 & Col de Dame Blanche & Faible & Instable & 3,0 & 3,0 & 3,0 & Étalé \\
\hline 23 & Conadria & Grande & Stable & 4,8 & 5,0 & 4,8 & Étalé \\
\hline 24 & Shahami & Faible & Stable & 3,8 & 4,0 & 3,6 & Étalé \\
\hline 25 & Shatawi & Grande & Stable & 5,0 & 4,0 & 5,0 & Étalé \\
\hline 26 & Tena & Grande & Stable & 4,8 & 4,8 & 4,8 & Érigé \\
\hline 27 & $\mathrm{~K}-4$ & Moyenne & Instable & 5,0 & 4,0 & 5,0 & Étalé \\
\hline \multicolumn{8}{|c|}{ Groupe des figues vert-violet } \\
\hline 1 & Gadol Adom & Moyenne & Instable & 4,8 & 5,0 & 4,8 & Érigé \\
\hline 2 & Zaraqi Kadoori & Moyenne & Stable & 3,5 & 3,5 & 3,5 & Étalé \\
\hline 3 & Hamari Beit Jann & Moyenne & Stable & 3,0 & 3,0 & 3,0 & Étalé \\
\hline 4 & Hamudi & Moyenne & Stable & 4,5 & 4,5 & 4,0 & Étalé \\
\hline 5 & Tu'ali Shehora & Faible & Stable & 3,5 & 3,5 & 3,5 & Érigé \\
\hline 6 & $\mathrm{~K}-16$ & Moyenne & Stable & 4,0 & 4,0 & 4,5 & Érigé \\
\hline 7 & Mu’azi & Faible & Instable & 3,0 & 3,0 & 4,0 & Moyen \\
\hline 8 & Mission & Moyenne & Stable & 4,0 & 4,0 & 4,5 & Érigé \\
\hline 9 & Saba'i & Moyenne & Instable & 4,0 & 4,5 & 4,0 & Érigé \\
\hline 10 & Asali & Moyenne & Stable & 4,5 & 4,5 & 4,5 & Étalé \\
\hline 11 & Flanders & Moyenne & Instable & 4,0 & 4,5 & 4,5 & Érigé \\
\hline 12 & Cope & Moyenne & Stable & 4,5 & 5,0 & 4,8 & Étalé \\
\hline 13 & Karak'i & Faible & Instable & 4,0 & 3,8 & 4,0 & Étalé \\
\hline 14 & $\mathrm{P}-20$ & Grande & Stable & 4,0 & 4,0 & 4,5 & Érigé \\
\hline \multicolumn{8}{|c|}{ Groupe des figues brunes } \\
\hline 1 & Ghazali & Faible & Instable & 4,5 & 4,8 & 4,5 & Érigé \\
\hline 2 & Khurtamani & Faible & Stable & 4,5 & 5,0 & 4,5 & Érigé \\
\hline 3 & Hamadi & Moyenne & Stable & 4,5 & 4,5 & 4,3 & Érigé \\
\hline
\end{tabular}




\begin{tabular}{|c|c|c|c|c|c|c|c|}
\hline $\mathrm{N}^{\circ}$ & Variété & Productivité & $\begin{array}{c}\text { Stabilité } \\
\text { des récoltes }\end{array}$ & $\begin{array}{c}\text { Quantité } \\
\text { de pousses }{ }^{1}\end{array}$ & $\begin{array}{l}\text { Longueur } \\
\text { de pousses }{ }^{1}\end{array}$ & $\begin{array}{c}\text { Taille } \\
\text { des arbres }^{1}\end{array}$ & $\begin{array}{c}\text { Port } \\
\text { des arbres }\end{array}$ \\
\hline \multicolumn{8}{|c|}{ Groupe des figues noires } \\
\hline 1 & Adriatica & Grande & Stable & 4,5 & 4,0 & 4,5 & Moyen \\
\hline 2 & Italqit Shehora & Moyenne & Stable & 4,5 & 4,5 & 5,0 & Érigé \\
\hline 3 & Diehl Noire & Moyenne & Stable & 4,5 & 4,8 & 4,3 & Érigé \\
\hline 4 & Harubi & Moyenne & Stable & 4,3 & 4,2 & 4,3 & Étalé \\
\hline 5 & Yoqne'am Shehora & Faible & Stable & 3,5 & 3,5 & 3,5 & Étalé \\
\hline 6 & Mat 1 & Moyenne & Alternante & 4,5 & 4,5 & 4,5 & Érigé \\
\hline 7 & Su'adi & Moyenne & Stable & 4,5 & 4,5 & 4,5 & Étalé \\
\hline 8 & Col de Dame Noire & Faible & Instable & 4,0 & 4,0 & 3,8 & Érigé \\
\hline 9 & Robine & Grande & Stable & 4,5 & 4,0 & 5,0 & Étalé \\
\hline 10 & Shaltufat Al-Abed & Moyenne & Stable & 4,5 & 4,0 & 4,5 & Érigé \\
\hline
\end{tabular}

De même, les variétés Biyadi, Italiqit Shehora (noire) et leurs clones ont un port dressé et des feuilles identiques.

Les clones de la variété Harubi ont été déterminés à partir des caractères de leur fruit, mais chacun d'eux se distingue par un port de l'arbre différent : étalé chez Harubi Ramallah et Mevo Huron, semi-étalé chez Porat Shehora et dressé chez Bet Guvrin Shehora. La feuille est identique dans toutes ces variétés et leurs clones.

\section{6. Économie agricole du figuier}

\subsection{Généralités}

En Israël et dans beaucoup de pays méditerranéens, il existe très peu de vergers de figuiers bien ordonnés, présentant des arbres plantés en lignes et conduits avec de bonnes techniques culturales. Le marché porte sur environ 1000 t de figues commercialisées en produit frais pendant les 2 mois d'été (juillet, août) et quelques jours en septembre. Le fruit se vend à très bon prix, dépassant 1,5 US\$ le kg. De consommation très agréable, juteux avec un goût de miel, il est très apprécié comme fruit frais, surtout celui issu de la production des arbres de montagne et des collines de Galilée et de Judée. La plus grande partie de la récolte se vend le long des routes; une faible partie de la production est commercialisée dans les magasins et les supermarchés. Le plus grand défaut de ce fruit est sa brève et faible tenue sur les étalages de vente et sa fragilité au transport.

La rentabilité du figuier étant peu satisfaisante [14, 15], la plantation de figuiers est limitée. La récolte se fait manuellement; elle est délicate, pénible et exige des passages répétés tous les trois jours. Elle occasionne des frais très élevés. Les fruits ramassés sont relativement petits, légers, cachés entre les feuilles, désagréables au toucher. Ils sécrètent à la coupe un latex blanc, collant, qui irrite la peau. À la cueillette, au triage, à l'emballage et au transport, le fruit est très sensible aux coups, surtout lors des journées chaudes d'été.

Les cultivars à fruit allongé, peau épaisse, ostiole fermé, ne s'éclatant pas et ayant une bonne tenue à l'étalage sont les plus intéressants à cultiver [14-16]. La variété «Madeleine des quatre saisons »cultivée en France a ces caractéristiques. C'est une variété bifère à récolte stable de figues-fleurs et récolte de figues d'été intéressantes et bonnes qui mûrissent 1,5 mois après les figues-fleurs. Deux autres cultivars, «Goutte 
d'or » et «Longue d'août », lui ressemblent [16].

Lorsque les modalités de croissance et de fructification d'une variété sont connues, les passages de cueillettes peuvent être réduits à trois ou quatre. Cela est obtenu en effectuant des tailles d'hiver et d'été destinées à induire une production groupée par raccourcissement des branches, comme cela se fait en Californie, en France et dans d'autres pays [7-13].

Les techniques de conditionnement des fruits ont été bien améliorées au cours des dernières années. Des caisses en carton, de taille moyenne avec des alvéoles en plastique permettent, comme pour les nectarines, d'individualiser chaque fruit. Cela permet aux figues de conserver une bonne présentation

D'un point de vue économique, un verger adulte possédant de grands arbres d'une variété unifère permet de récolter environ $40 \mathrm{t} \times \mathrm{ha}^{-1}$, ce qui conduit à un revenu brut de 50000 US $\$ \times \mathrm{ha}^{-1} \times \mathrm{an}^{-1}$. Les frais imputables à la culture et à la récolte atteignent environ $50 \%$ du revenu brut. La production pourrait donc être rentable, mais le travail nécessaire au maintien d'un beau fruit jusque sur les marchés augmente ce coût et diminue les recettes. Une meilleure organisation de la commercialisation, telle qu'elle existe dans les coopératives françaises, conduit à améliorer la présentation finale du fruit, donc à augmenter la demande tout en maintenant des prix plus avantageux [17].

Une expérimentation effectuée à Toulouse [16] a permis d'étudier l'effet de certains traitements des figues après récolte sur leur durée de vie. Les meilleures conditions de stockage pour conserver un fruit sain ont été obtenues en plaçant les fruits à $2{ }^{\circ} \mathrm{C}$ durant une courte période. Par ailleurs, le fruit se conserve bien à une température de $8{ }^{\circ} \mathrm{C}$, pendant $10 \mathrm{j}$. Le maintien d'une atmosphère contrôlée à $20 \%$ de $\mathrm{CO}_{2}$ dans les installations frigorifiques a donné de bons résultats de conservation. Des traitements, avant stockage au froid, consistant à placer au fond du carton d'emballage du papier imbibé avec des concentrations faibles de $\mathrm{SO}_{2}$ ou de $\mathrm{N}_{2} \mathrm{O}$ pour éviter le blanchiment du fruit, semblent être très prometteurs, surtout si le carton de condition-nement est enveloppé d'un film de polyéthylène. Ces recherches se poursuivent et permettront peut-être de compléter ces premiers résultats [16]. Dans les vergers ordonnés, il est possible d'utiliser aujourd'hui des machines de calibrage manuel qui abîment très peu le fruit.

L'utilisation d'hormones qui remplacent la fécondation des variétés femelle représente un progrès important : le traitement de l'arbre par des pulvérisations d'acide parachlorophénoxyacétique à 80 ppm s'effectue pendant la période de la caprification et donne d'excellents résultats. Les fruits obtenus sont sans graines, parthénocarpiques ; ils mûrissent plus tôt, sont de plus gros calibre et d'une bonne qualité gustative [1].

\subsection{Les figues-fleurs}

Les producteurs de figues se sont toujours intéressés à la production de figues-fleurs. Les prix de gros que ces fruits peuvent atteindre sur les marchés - jusqu'à $6 \mathrm{US} \$ \times \mathrm{kg}^{-1}$ - sont très rémunérateurs et il y a donc un grand intérêt à développer cette culture. Au cours de nos travaux, le clone Nazareth qui a été sélectionné s'est révélé le plus intéressant; il donne une très grande figue-fleur, vert-jaune et bonne. Sa figue est vendue à des prix avantageux pour le producteur ces prix peuvent atteindre $10 \mathrm{US} \$ \times \mathrm{kg}^{-1}$, soit environ $70 \mathrm{FF} \times$ $\mathrm{kg}^{-1}$, pour les premiers fruits récoltés à la mi-avril. Avec la production enregistrée de 6 à $10 \mathrm{t} \times \mathrm{ha}^{-1}$ de fruits, la culture devient rentable.

La deuxième récolte, ou récolte d'été, est toujours plus importante que cette première récolte ; elle peut atteindre un tonnage quatre fois supérieur. Les fruits sont assez précoces et de grande qualité : relativement gros, jaune-doré, doux (28\% de solides solubles totaux), très juteux avec un goût de miel et parfumés.

De nombreuses recherches se sont intéressées à la production des figues-fleurs. Un travail de base est dû à Rivals dans les années 70 ; il a permis de déterminer le 
processus de leur formation sur les différents rameaux des arbres [5, 7, 9, 10]. Le pincement pré-printanier des branches a donné de bons résultats. Des traitements hormonaux et l'incision annulaire testés lors de ces travaux n'ont pas eu d'effet [5, 7, 9, 10]. Au début des années 1980, une équipe de chercheurs israéliens a entrepris une étude sur ce sujet. Tout d'abord, les récoltes obtenues se sont révélées non stables et les fruits ont été de mauvaise qualité. Par la suite, plusieurs problèmes ont été résolus ; cela a permis de meilleures récoltes dont une partie a pu être exportée $[11,12]$. De nouvelles plantations ont été mises en place, mais elles ont été ensuite en partie abandonnées car la qualité des fruits obtenus est encore insuffisante malgré les très gros fruits récoltés.

Depuis, les vergers de Nazareth, encore restreints il y a quelques années (40 ha), ont été agrandis et presque doublés dans la vallée du Jourdain au cours des quatre dernières années ; ils ont permis de réaliser de très bonnes recettes gràce à la mise au point de nouvelles techniques. Ainsi, une pulvérisation hivernale, au cyanamide de calcium acidulé $\left(\mathrm{H}_{2} \mathrm{Cn}_{2}\right.$, Alzodef), de figuiers de la variété sélectionnée Nazareth améliore le débourrement et diminue la chute des figues-fleurs. L'acide gibbérellique $\left(\mathrm{GA}_{3}\right)$, appliqué dans un second temps, a un effet encore plus accentué sur cette chute des fruits, sur la croissance des ébauches et sur leur départ en végétation. De même, il diminue la chute des ébauches de figues formées en automne. Un traitement au benzyladénine diminue la chute de la dernière vague de figues-fleurs au printemps [11, 12]. Quelques nouveaux essais se pratiquent sur d'autres variétés. À Montpellier (France), pour augmenter les récoltes de figues-fleurs, des techniques culturales accompagnés de tailles appropriées sont préconisées [8]. Des arbres très ramifiés présentant des branches courtes de 7 à 11 entre-nœuds, les plus productives, sont formés et un court arrêt de l'irrigation en été favorise également la production.

En Israël, certaines variétés en provenance de France (Conservatoire botanique, Centre de Porquerolles) sont en cours d'introduction à titre expérimental ; elles sont surtout du groupe San-Pedro, bifères $[4,18]$. D'autres variétés de Turquie, d'Italie, de Chypre et de Grèce sont également en cours d'évaluation. Tous les traitements développés pour la bonne récolte de figuesfleurs sont mis en œuvre.

Pendant la dernière saison de l'an 2000, pour la première fois dans la station de recherches de Néwé-Ya'ar, certaines variétés anciennes parmi lesquelles Dafur de Ramallah, K-4 ou Porat Yeruqqa, placées en bonnes conditions de culture, ont donné des figues-fleurs. Ces variétés seront expérimentées dans d'autres sites.

Les figues-fleurs produites par des variétés différentes ont une durée de croissance du fruit et une date de maturité différente. Cette particularité peut être exploitée pour allonger la période de récolte au-delà de 2 mois et donc approvisionner les marchés plus longtemps. Les variétés dont la durée de croissance du fruit est longue sont aussi de meilleure qualité gustative.

En Israël, les premières figues de la variété Nazareth sont récoltées dans la vallée du Jourdain, très chaude, à la fin du mois d'avril, mais elles subissent d'importantes attaques de la mouche du figuier et leur qualité est médiocre. Dans les vallées intérieures chaudes d'Israël - vallées Izreel et Kefar Tabor - et sur la plaine côtière, la maturité intervient au début du mois de mai ; les fruits sont de qualité moyenne et les attaques de mouches moyennes à faibles. Dans la Moyenne et Haute Galilée, les figues sont récoltées au début du mois de juin ; leur qualité gustative est très bonne ; les fruits sont gros et ne subissent presque pas de dégâts par les mouches du figuier. Pour cette raison, en Israël et dans les pays méditerranéens chauds, deux méthodes de plantation sont préconisées pour récolter des figues-fleurs pendant une longue saison :

- La première méthode consiste à cultiver, dans une même région, une gamme de variétés à figues-fleurs qui, en faisant se succéder leur période de récolte, permettront une longue époque de cueillette. Ainsi, par exemple, dans la vallée du Jourdain, les variétés Nazareth et Dauphine produiront pendant 1,5 mois. D’autres variétés 
plus tardives, telles que Madeleine des quatre saisons et Banane, pourraient, d'après les observations faites au centre de Porquerolles (France), prolonger la saison de récolte d'un mois supplémentaire.

- La deuxième méthode consiste à planter dans différentes régions, sous différents climats, une bonne variété telle que Nazareth qui donnera des fruits pendant 3 mois, les premières récoltes se faisant en régions chaudes et les plus tardives en régions froides de haute montagne.

La culture biologique sous filet de variétés bifères aptes à produire une grande récolte de figues-fleurs, entreprise au cours de ces dernières années, semble être très prometteuse. De nouveaux problèmes touchant la différenciation des boutons floraux et les attaques des acariens, observées dans ces conditions particulières de culture, sont cependant à résoudre.

\section{Techniques de culture recommandées}

Les résultats obtenus à l'issue de plusieurs essais spécifiques, accompagnés par des analyses de sol, de feuilles et des mouvements d'eau dans le sol, permettent de recommander des techniques de cultures adaptées à la production de figues en Israël.

\subsection{Plantation}

La plantation s'effectue l'hiver par des boutures épaisses de bois de 1 an, de préférence à talon, longues de 25 à $30 \mathrm{~cm}$, à raison de deux à trois boutures enterrées à chaque emplacement d'arbre. Après avoir tassé un peu le sol, les boutures sont recouvertes d'au moins $15 \mathrm{~cm}$ de terre. Une irrigation effectuée de temps en temps doit permettre de maintenir une bonne humidité dans le sol. La réussite est alors de $100 \%$ et, au bout de 2 mois, la nouvelle pousse de 1 à $2 \mathrm{~m}$ obtenue est rabattue et raccourcie à $30 \mathrm{~cm}$.

Les distances de plantation recommandées vont de 4,5 à $5 \mathrm{~m}$ entre les rangées et de 3 à $4 \mathrm{~m}$ entre les arbres. Des plantations plus serrées n'ont pas donné de bons résultats.

\subsection{Taille}

Les arbres doivent être menés en gobelet bas très ouvert qui correspond à la structure la plus économique. Le tronc court, de $30 \mathrm{~cm}$, a alors quatre à cinq charpentières très basses. Une grande ramification et l'étalement des arbres sont obtenus par des pincements effectués au printemps et en été, lors de la première et de la deuxième année. Cela permet de pouvoir ensuite effectuer la récolte du sol. Dans certains cas, il est nécessaire d'ouvrir les arbres en attachant les branches entre elles ou à des crochets implantés au sol.

La taille classique des arbres adultes en hiver se fait par élagages de branches surtout dans le centre de l'arbre. Les rameaux sont rabattus et raccourcis pour rajeunir les arbres, obtenir de nouvelles pousses et regrouper la récolte.

\subsection{Irrigation et fertilisation}

Dans les jeunes plantations, les irrigations et les fertilisations doivent être appliquées très fréquemment et en relativement grandes quantités. Le figuier répondant à ces traitements par une excellente pousse, cela permet de ramasser des fruits dès la deuxième année et de disposer d'arbres couvrant toute la surface du sol dès la quatrième année, avec de grandes et pleines récoltes. La fertirrigation est en général journalière pour les jeunes arbres, puis elle passe à deux fois par semaine.

Dans un verger adulte, l'irrigation et la fertilisation sont modestes : entre le tiers et la moitié de la dose normale apportée aux autres arbres fruitiers. Les quantités d'eau apportées sont autour de 0,3-0,4 fois l'évapotranspiration (ou du Pan Class A Evaporation) mesurée habituellement dans les vergers israéliens. La fertirrigation doit être préférentiellement basée sur le système du goutte-à-goutte avec, par rangée, une seule rampe de goutteurs de $4 \mathrm{~L} \times \mathrm{h}^{-1}$ placés tous les mètres. En principe, toute l'eau est apportée par des solutions nutritives dont la composition est déterminée à partir des besoins des arbres et de la récolte. Cellesci doivent fournir, pour toute la saison, autour de $150 \mathrm{U} \times$ ha $^{-1}$ d'azote et environ 
$300 \mathrm{U} \times \mathrm{ha}^{-1}$ de potasse. Les autres éléments nutritifs sont apportés suivant les besoins, la végétation et l'aspect des feuilles. Un mois à un mois et demi avant la récolte, il faut réduire de beaucoup la fertirrigation et ne pas appliquer de fertilisants. Les quantités d'eau doivent alors se situer autour de 0,2-0,3 fois l'évapotranspiration totale, pour obtenir une bonne maturité des fruits, un fruit sucré, mielleux et parfumé.

\subsection{Récolte}

Les opérations liées à la récolte sont coûteuses. Elles nécessitent deux ou trois passages par semaine, pendant deux ou trois semaines, selon la variété. Le fruit est très fragile, sa peau se déchire facilement et s'abîme. La cueillette n'est pas agréable à cause du latex collant et irritant, produit au niveau de la coupe de la figue. Afin de limiter les manipulations, les fruits doivent être ramassés fermes, mûrs, puis placés directement en plateaux de vente munis d'alvéoles, de préférence, ou en barquettes. Il existe plusieurs techniques d'aide à la cueillette que chaque producteur développe et adopte pour limiter les frais.

Des trieuses de fruits perfectionnées peuvent être utilisées ; l'emballage est alors, en général, constitué de plateaux de carton contenant une seule couche d'alvéoles. Cette opération doit être effectuée immédiatement après la récolte et avec beaucoup de précautions pour approvisionner le marché avec un fruit de qualité, bien présenté et pouvant donc atteindre un prix intéressant. Une chaîne de froid intervenant directement après la cueillette facilite le travail.

\section{Conclusions et discussion}

Pour les producteurs de pays développés tels que la France, l'Italie et l'Espagne, la culture du figuier est rentable et prometteuse. La demande de fruits de qualité est en hausse et l'avenir de cette culture est grand pour les marchés locaux et, surtout, pour l'exportation. C'est un fruit apprécié par l'ensemble des populations méditerranéennes et, actuellement, les prix sur les marchés sont élevés.

Les figues commercialisées en France sont minutieusement triées et emballées, ce qui leur confère une bonne durée de vie sur les étalages. Ces fruits ont une très bonne présentation et sont de bonne qualité. Cette amélioration des conditions de vente se propage dans tous les pays méditerranéens, dont Israël.

Pour réussir la culture de la figue d'été et d'automne, il faut planter trois à quatre variétés parmi les meilleures en cherchant à étaler la récolte sur toute la période où la demande existe. La classification, l'étude des caractères et des performances de tout le matériel végétal qui a été collecté en Israël au cours de la deuxième moitié du vingtième siècle permet aujourd'hui de proposer un choix parmi les variétés à exploiter et de donner des conseils de culture aux exploitants.

Les techniques particulières à apporter à la culture de variétés donnant des figuesfleurs justifient l'implantation de parcelles à part dans le verger. À l'issue des travaux effectués, un certain nombre de type de figuiers appartenant aux quatre groupes qui ont été précédemment identifiés peuvent être recommandé pour la production de récoltes d'été et d'automne :

- groupe des figues vert-jaune : Kefar Uriyya, Yafo Kadoori, Kurt, Smyrna et Shahamani, la plus belle variété des types Nazareth ;

- groupe des figues vert-violet: Gadol Adom, Hamudi et Saba'i ;

- groupe des figues brunes : Khurtamani et Ghazali qui est le meilleur clone ;

- groupe des figues noires : Italqit Shehora, Harubi, Robine et Shaltufat Al-Abed.

La variété Nazareth cultivée avec les techniques appropriées qui ont été exposées est la plus recommandée pour une production préférentielle de figues-fleurs.

À la condition d'apporter des soins de culture adaptés à l'exploitation de ces meilleures variétés de figuier, la filière «figue »pourrait devenir une branche attractive et rémunératrice en Israël. 


\section{Note}

L'auteur dirige depuis 35 ans la recherche sur le figuier en Israël. Il fait partie de la direction nationale des producteurs de la figue en Israël et de ce fait il participe à tous les travaux de développement.

\section{Remerciements}

L'auteur remercie vivement les très nombreuses personnes qui ont contribué à collecter le matériel végétal, à réaliser et à planter les parcelles et à accumuler les données à Kefar Hanania ; aux techniciens de vulgarisation de la Galilée, et particulièrement à M. Adawi S. ; aux directeurs successifs de la station expérimentale et surtout à M. Ran I. Sa reconnaissance va également aux techniciens du Centre de recherches de Néwé-Ya'ar et surtout à Mme Bar-Yaacov I. qui a participé aux travaux d'appréciations et de classification des variétés et à M. Hatib Camel.

\section{Références}

[1] Goor A., The fig, Ministry of Agriculture, Agricultural Publication Section, Tel Aviv, Israël, 1955, $67 \mathrm{p}$.

[2] Condit I.J., Fig varieties, Hilgardia 23 (1955) 323-538.

[3] Condit I. J., A bibliography of the fig, Hilgardia 25 (1956) 1-663.

[4] Anonyme, Le figuier Ficus carica L., Conservatoire botanique de Porquerolles, Pocquerolles, France, 1994, 30 p.

[5] Rivals P., Notes biologiques sur le figuier, J. Agric. Trop. Bot. Appl. IX (1-3) (1962) 55-64.
[6] Assaf R., Studies on shoot growth of different fruit species, J. Agric. Trop. Bot. Appl. 13 (4-5) (1966) 147-182.

[7] Rivals P., Karabiyik N., Considérations sur les figuiers bifères et sur la production des figues-fleurs, Agron. Lusitanica 32 (Oct.) (1971) 173-182.

[8] Lauri P.-É., Caraglio Y., Figuier. Optimiser la production de figues-fleurs, Arboriculture Fruitière 489 (1995) 25-28.

[9] Rivals P., Sur les moyens naturels susceptibles d'accroîte la production des figuesfleurs, Fruits 33 (2) (1978) 117-119.

[10] Rivals P., Sur les variations de la précocité et du déroulement des maturations chez les figuiers, Fruits 34 (3) (1979) 199-202.

[11] Weisman Z., Erez A., Goor A., Shulman Y., Brebas figs as a commercial culture, Part A, Hassade 66 (3) (1985) 503-505.

[12] Weisman Z., Erez A., Goor A., Brebas figs as a commercial culture, Part B, Hassade 66 (3) (1985) 506-512.

[13] Assaf R., Genetic bank of deciduous fruit trees and grapes, official lists of ARO, Special publication for the Israel Academy of Science and Development, ARO, Névé Ya'ar, Israël, 1992, 8 p.

[14] Brunner C., Figues. De grandes espérances, Arboriculture Fruitière 488 (1995) 39-43.

[15] Carles L., Le figuier. Précautions à prendre pour sa culture, Arboriculture Fruitière 374 (1985) 37-38.

[16] Baccaunaud M., Raynel J., Baboulene F., Figues. Maintien de la qualité après récolte, Arboriculture Fruitière 489 (1995) 37-42.

[17] Anonyme, Figue en marge de la filière, Fruits et Légumes 123 (1994) 28-29.

[18] Anonyme, Listes des cultivars plantés au Conservatoire botanique de Porquerolles, J.P. Roger (éd.), Porquerolles, France, 1992, $8 \mathrm{p}$.

\section{Selección de variedades locales y técnicas de cultivo de la higuera en Israel.}

Resumen - Introducción. En Israel, el higo es un fruto muy consumido. El artículo recapitula acerca de las nuevas técnicas probadas y adoptadas para el cultivo de la higuera y presenta los resultados de ensayos varietales efectuados durante 50 años en huertos frutales sucesivos de estaciones experimentales, compuestos de un gran número de variedades provenientes de una amplia recolección realizada a través de Israel. Generalidades. Tras recordar el 
origen de la higuera, se describen las particularidades de la flor y su fecundación, o cabrahigadura, mediante Blastophaga psenes. La higuera puede ser unífera y producir una cosecha al año en otoño, o bífera y fructificar dos veces: en primavera (brevas) y en verano-otoño (higos). Se describen brevemente los mecanismos de crecimiento y fructificación del árbol. Material y métodos. El estudio realizado apuntó a la producción de huertos experimentales creados en Alta Galilea (Israel) y a la observación de la producción de higueras recolectadas en todo el país, Gaza, Sinaí y Golán. Resultados y clasificación de las variedades estudiadas. La clasificación de las variedades de higos se fundamenta primeramente en las características básicas del fruto y, después, en observaciones complementarias. Se diferenciaron, globalmente, cuatro grupos de higueras a partir del color verde-amarillo, verde-violeta, pardo-estriado y negro de los higos. Cada una de las variedades identificadas en dichos grupos fue descrita, se analizó el crecimiento de los árboles considerando también la economía agrícola de la higuera. Se exponen las técnicas más adecuadas para el manejo del cultivo de la higuera desde su siembra hasta la cosecha. Conclusiones y discusión. Al término de los trabajos realizados, algunos de los cuatro tipos de higueras identificados son particularmente recomendados. La variedad Nazareth se aconseja para la producción de brevas.

Israel / Ficus carica / selección / variedades / cultivo 